\title{
Rehabilitating Psoas Tendonitis: A Case Report
}

\author{
Jaime Edelstein, PT, MSPT, CSCS
}

Received: 21 August 2008/Accepted: 14 October 2008/Published online: 2 December 2008

(C) Hospital for Special Surgery 2008

\begin{abstract}
This case report describes the examination and physical therapy intervention for a woman with anterior hip pain whose medical diagnosis following magnetic resonance imaging (MRI) was bilateral labral tears and psoas tendinitis. Her physical therapy evaluation revealed findings consistent with psoas tendonitis. Utilizing theories of neuromuscular patterning and knowledge of normal muscle function, the patient was successfully treated in physical therapy following six physical therapy sessions, once a week for 6 weeks. The patient was found to have an overactive psoas muscle, as indicated by hip flexion being the primary mover in her movement patterns, and dysfunctional abdominal and pelvic floor muscles. Functionally based therapeutic exercise and electrical stimulation were used to reeducate the muscles of the abdomen, pelvic floor, and hips in order to create muscular balance and correct muscle dysfunction.
\end{abstract}

Keywords psoas tendonitis · hip labrum .

neuromuscular re-education

\section{Introduction}

It is not uncommon for patients to be referred to physical therapy with a diagnosis of a hip labral tear and a prescription for "hip flexor stretching" for the psoas muscle. The clinical basis for this referral is the assumption that the psoas must be part of what is causing the pain and therefore must be stretched. The physical therapist must then determine through proper examination and assessment what role the psoas muscle and other soft tissue structures have in this pathological process. However, in the author's experience, physical examination of these patients often confirms that the psoas is not short or tight, but rather lengthened and

J. Edelstein, PT, MSPT, CSCS $(\bowtie)$

Hospital for Special Surgery,

Sports Rehabilitation and Performance Center,

535 East 70th Street, New York, NY 10021, USA

e-mail: edelsteinj@hss.edu overused. Therefore, additional stretching of this dysfunctional muscle is not useful in the physical therapy treatment plan and may lead to additional pain if the muscle and tendon is hypersensitive. Physical therapists routinely receive prescriptions requesting treatment to stretch, strengthen, and stimulate muscles. In some cases, muscles are overstimulated and need therapy to inhibit them through exercise and movements. In this case, "overstimulated" means the muscle is being called upon to function in movement patterns exceeding its normal function and therefore creates dysfunctional movement patterns and potential pathology and pain. The muscle is always "on," functioning both in expected movement patterns and, when not expected, leading to faulty movement patterns. The process of identifying an overused and overstimulated muscle is a combination of clinical observation of movement patterns, and the muscle is often tender to palpation. Once the muscle is inhibited and relieved of its overly stimulated state, only then may it be functionally reeducated.

Dr. Vladimir Janda, a Czech neurologist and physiatrist, devoted his career to investigating muscle imbalance syndromes and the means to treat them. Janda took the functional approach in assessing chronic musculoskeletal pain, whereby all systems of the body were recognized and examined versus just focusing on the acute region of pain [1]. Janda focused his research on examining the association of the musculoskeletal and central nervous systems and how muscles respond to the sensorimotor system. It has been well recognized that muscles may be stimulated, inhibited, or changed in tone based on a response to pain or altered proprioceptive input. Studies have repeatedly proven this phenomenon to be accurate [2-4]. Panjabi, like Janda, believed that the subsystems are functionally interdependent and develop adaptations and compensations to maintain homeostasis within the whole system [5]. Vladimir Janda identified muscles as either functionally tonic or phasic [6]. The tonic muscles are often the flexors and are phylogenetically older and dominant. The phasic system consists of extensors and is developed shortly after birth. Janda noticed that muscular imbalances may be the result of neurological 


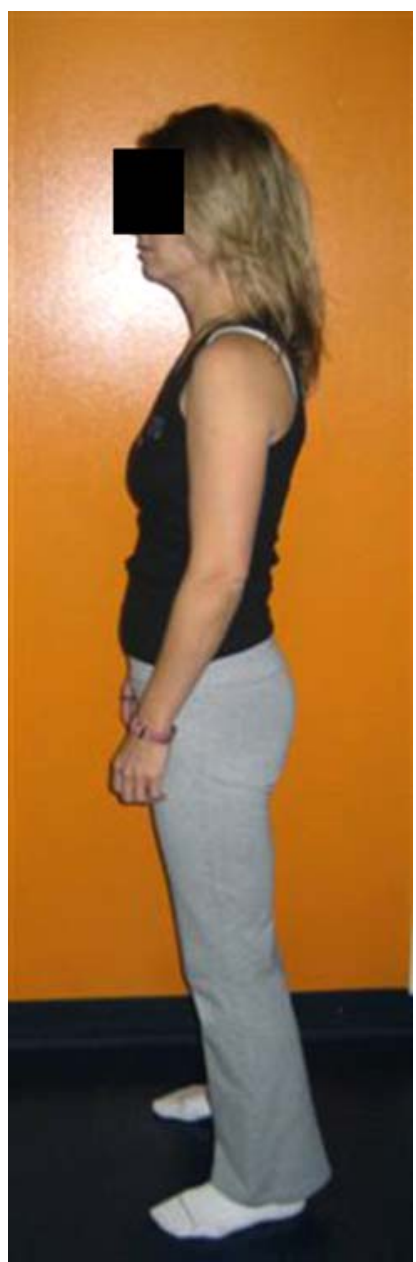

Fig. 1. The subject's initial posture displays a sway back and posterior pelvic tilt

changes, not only structural changes in the muscle $[1,6]$. Being able to recognize these patterns of imbalance due to inhibition, overstimulation, tightness, or excess length becomes quite useful in the diagnosis as well as treatment of painful muscle syndromes similar to that evident in this patient.

\section{Case report}

A 43-year-old female was referred to physical therapy with a diagnosis of bilateral hip labral tears and psoas tendinitis. She worked full time in the entertainment industry and was on her feet many hours a day. She was an otherwise healthy individual who enjoyed gym workouts and being active with her two young children. During the initial subjective examination, the patient stated that she had popping and pain in both hips for 3 years, since 2004. However, when probing further into her medical history, it appeared that she recalled her hips "popping" when she was "very young." She was a hurdler on the track team in high school and remembered having a "bad back." She was diagnosed at that time with a lumbar disc herniation, which kept her from completing her track season. Her back pain subsequently resolved. She had only intermittent popping in her hips at that time. In 2004, the "popping" in both hips became worse. Assuming she needed more strength, she began taking a Pilates mat class. After 2 years of Pilates, she realized that the class was exacerbating her symptoms. The pain in her right hip was worse. The patient saw a physician in October 2006. Radiographs for the right hip were positive for an anterior acetabular bone spur. No radiograph was taken of her left hip. This patient was referred to physical therapy and initiated a program based on core strengthening and hip flexor stretching. Following 4 months of physical therapy, improvement was noted; however, the pain persisted. The patient ultimately sought medical attention at our institution. An MRI of both hips revealed right and left hip labral tears and psoas tendinitis. The patient was told that she would most likely require surgery to debride the labrum and to remove the acetabular bone spur. However, the physician wanted her to initiate physical therapy first. At the time of our evaluation, the patient's chief complaint was pain in both hips, right greater than left that woke her up out of her sleep if she was not positioned perfectly with pillows between her knees and if she did not lie in the correct back and hip position. She described pain $4 / 10$ on the visual analogue scale when sitting at her desk for more than $7 \mathrm{~min}$ and had pain while driving. She also described a need to "pop" her hips when she was walking.

During the physical examination, an observational gait analysis was performed as the patient walked from the waiting room into the gym. She presented with a significant sway back posture and a posterior tilt to her pelvis. (Fig. 1) She appeared to generate more rotation from her lumbar spine during gait than from rotation in her hips when transitioning from heel strike to mid-stance and then to push off. She demonstrated poor stability from her core during gait and advanced forward through hip flexion and maintained a constant sway back posture. The lumbar scan demonstrated full active range of motion and was otherwise unremarkable. All lumbar stress tests for torsion, shear, and compression were negative, and all sacroiliac joint stress tests were negative. Passive intervertebral movements (PIVMs) were unremarkable in the lumbar spine. Neurological tests for dural signs, sensation, deep tendon reflexes, and myotomal tests were all negative. Left hip range of motion was within normal limits and pain free. The right hip was within normal limits with anterior hip pain at end range hip flexion $\left(115^{\circ}\right)$ and end range internal rotation $\left(20^{\circ}\right)$. Strength measures and special tests were performed (Tables 1 and 2). The functional squat test was significant

Table 1 Strength testing

\begin{tabular}{llc}
\hline Strength testing & Right hip & Left hip \\
\hline Hip flexion & $4+/ 5$ with pain & $5 / 5$ \\
Hip extension & $5 / 5$ & $5 / 5$ \\
Hip abduction & $4-/ 5$ & $4 / 5$ \\
Hip adduction & $5 / 5$ & $5 / 5$ \\
Hip internal rotation & $5 / 5$ & $5 / 5$ \\
Hip external rotation & $4+/ 5$ with pain & $5 / 5$ \\
Abdominal strength & Sahrmann level E1 & \\
\hline
\end{tabular}


Table 2 Special tests

\begin{tabular}{lll}
\hline Special tests & Right hip & Left hip \\
\hline $\begin{array}{ll}\text { Hip scour } \\
\text { Hip impingement }\end{array}$ & $(-)$ & $(-)$ \\
$\quad(f l e x i o n$, IR, adduction) & $(+)$ & $(+)$ \\
$\begin{array}{l}\text { Snapping hip test (hip } \\
\quad \text { flexion/Abd/ER into } \\
\text { hip extension/add/IR) }\end{array}$ & $(+)$ & $(-)$ \\
Thomas test & $(+)$ rectus femoris, $(-)$ psoas & $(-)$ \\
\hline
\end{tabular}

*The Snapping Hip test performed in this manner is in particular sensitive to the psoas snapping over the anterior acetabulum.

for decreased core stability and utilization of the psoas as the primary mover as indicated by movement in the pelvis and lumbar spine during the squat activity. A relative hip flexion movement initiated the squat in both the descending direction and on the return to standing. The 8-in. forward step up and step down tests indicated decreased abdominal and hip abduction strength and stability. The patient was tender to palpation over the right anterior acetabulum and over the insertion of the psoas at the posterior aspect of the lesser trochanter.

The subjective and physical exam was indicative of a right hip labral tear and a hypersensitive, yet not shortened right psoas muscle. The plan for physical therapy intervention was to regain normal neuromuscular function of the psoas through initial inhibition, and then re-education of the psoas, back extensors, abdominals, and surrounding hip musculature to support the pelvic region.

The patient was seen for one treatment a week in physical therapy and provided with education to perform a home program every other day. During the initial session, week 1, the patient was instructed in two exercises and patient education. First, a psoas inhibition exercise was taught, whereby there was facilitation of quadriceps and tibialis anterior with coincident inhibition of the hamstrings and psoas in supine [1, 6, 7]. (Fig. 2) The patient lies supine in hook lying and is instructed to dorsiflex at the ankle and push through their quadriceps as if to gently slide up the mat. The challenge is to contract the quadriceps without activating the hamstrings. If done correctly, the tibialis anterior and quadriceps are activated, while the hamstrings and psoas are inhibited [7]. A trunk curl exercise was also performed, with the same positioning and neuromuscular patterning as the inhibition exercise. This exercise simply adds a small curl by elevating only the scapula off the mat. The purpose of this exercise was to begin to reeducate and activate the abdominals, transversus abdominus, and rectus abdominus as well as the pelvic floor. Patient education focused on how her chair was positioned at work with regard to hip angle. The patient reported wanting to purchase a new chair to provide her with better back support and more of an open hip angle. The patient arrived for her second week session, reporting no soreness from the exercises. She did also report a "loosening" sensation deep in her hips which almost felt unstable. Physical therapy treatment consisted of a warm up on bike, followed by exercises as in session one. In addition, electric stimulation was used to initiate reeducation of the psoas in a functional manner [7]. (Fig. 3) The premise behind this exercise was to assimilate a walking pattern, but with the patient lying prone. The machine was set for muscle reeducation with the duty cycle set for $10 \mathrm{~s}$ on, $30 \mathrm{~s}$ off over the multifidus on the affected side. The patient was lying in prone with a pillow under her chest and pillow under her ipsilateral thigh. Directions to patient were to "push into the pillow with your knee when you feel the electric stimulation on your back." This was done for $7 \mathrm{~min}$ on each side. The follow-up exercise was for the patient to stand up and stride with verbal cues for neutral posture. This was done again to activate the psoas in a functional manner. The striding was added to the patient's home program.

The patient arrived for her third visit on week 3 reporting that she was able to get a better chair for work and had been consistent with her exercises at home. She reported a $60 \%$ improvement in her symptoms already. She felt better than she had felt in the last 2 years. Physical therapy included continued use of electric stimulation for psoas muscle reeducation followed by gait training with postural control as well as continuation of all therapeutic exercise. The Pilates Reformer was added this visit for the

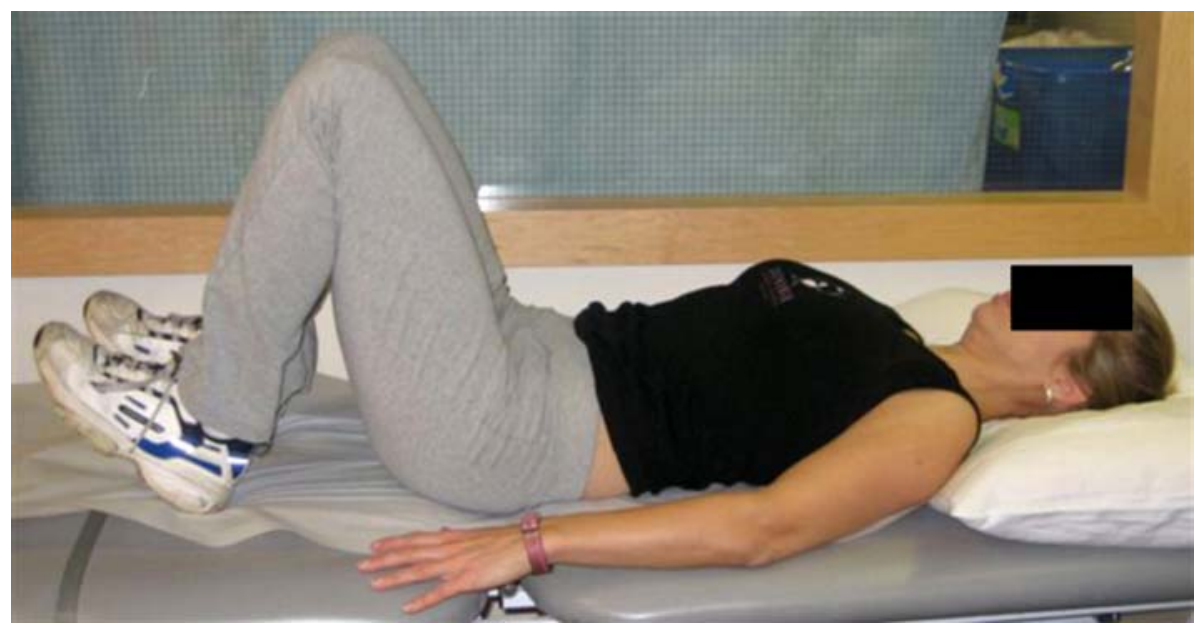

Fig. 2. The psoas muscle inhibitory exercise 


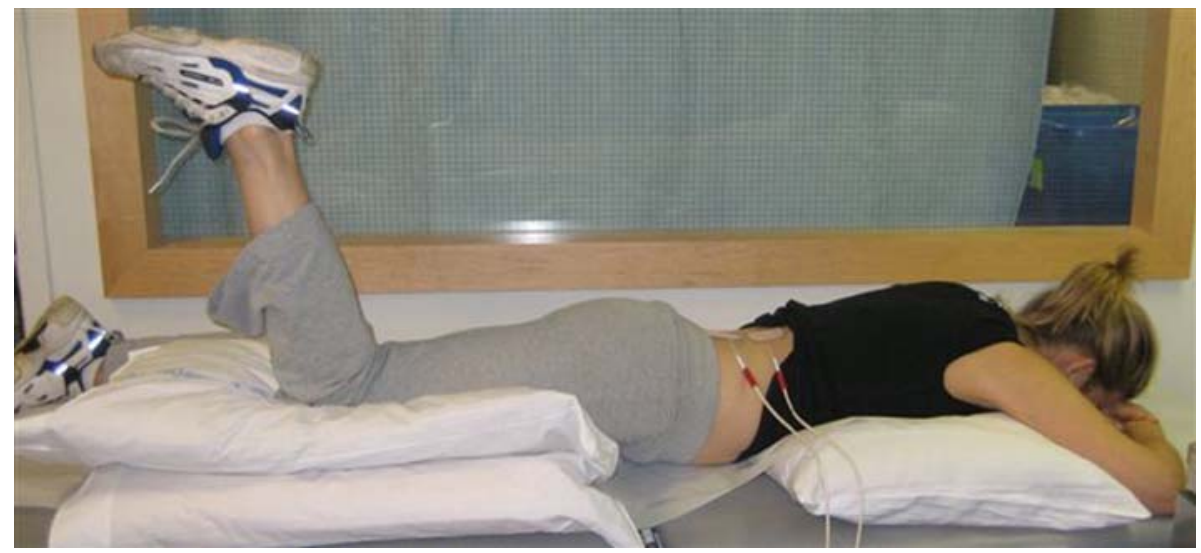

Fig. 3. Electric stimulation of the multifidus is performed for muscle reeducation of the ipsilateral psoas muscle

full foot series to be performed. This type of exercise encourages functional core control and stability while moving from the lower extremities. Although the patient did not have access to a Reformer outside of physical therapy, it was a great transitory tool for leading into other functional exercises including squats. The patient reported on the fourth visit, week 4 , that she did not have any additional hip pain from her last session, but her abdominals were sore, potentially from the Reformer exercise. The program was not changed for this visit. On the fifth visit, the patient reported a $75 \%$ improvement in her symptoms with complete resolution of her left hip pain. However, she still had right anterior hip pain when she wore high heels. During this session, the Psoas Bum Walk exercise was added, which she performed successfully for a 6-in. distance. (Fig. 4) The psoas walk is an exercise developed by Erl Pettman, based on Gracovetsky's work in observing a thalidamine victim and then his subsequent papers examining the use of the spine, pelvis, and hips in locomotion $[7,8]$. The theory behind this exercise is again to promote isolated strengthening of the psoas muscle. The patient sits long on the floor and advances themselves forward using only the psoas muscle. The patient's hands may either be in their lap or across their chest, as long as

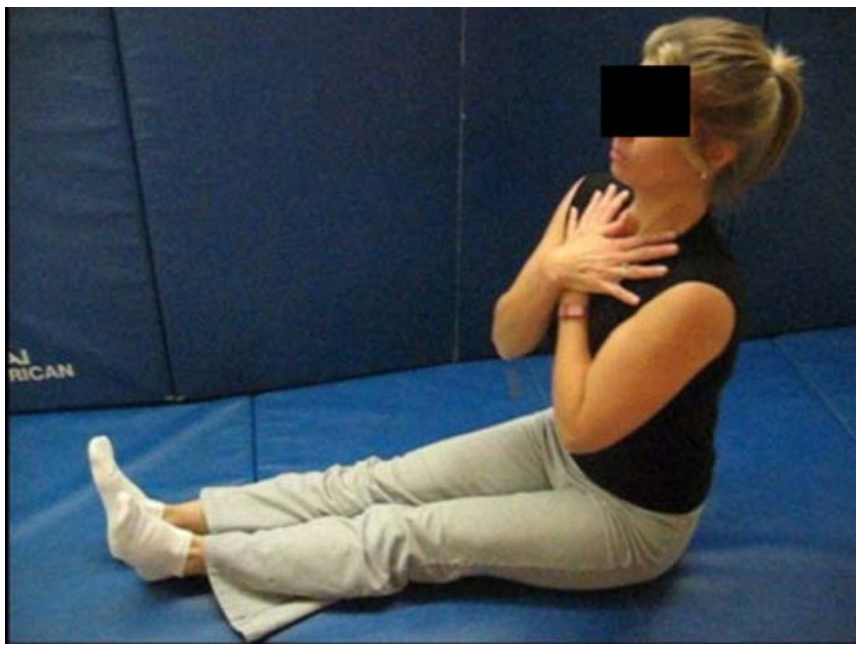

Fig. 4. The Psoas Bum Walk exercise is demonstrated they are not used to assist the motion. The patient is given verbal cues to advance forward, alternating movement from their right buttock to their left, without laterally shifting weight. This exercise was added to her home program. During her final session on week six, the patient reported focusing greatly on her standing and walking posture. She had noticed abdominal muscle soreness with focus on her

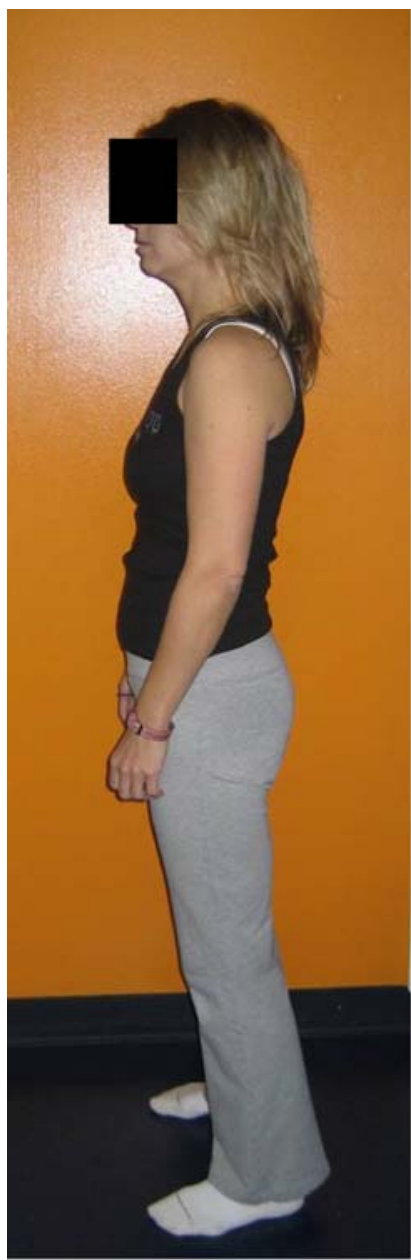

Initial Exam

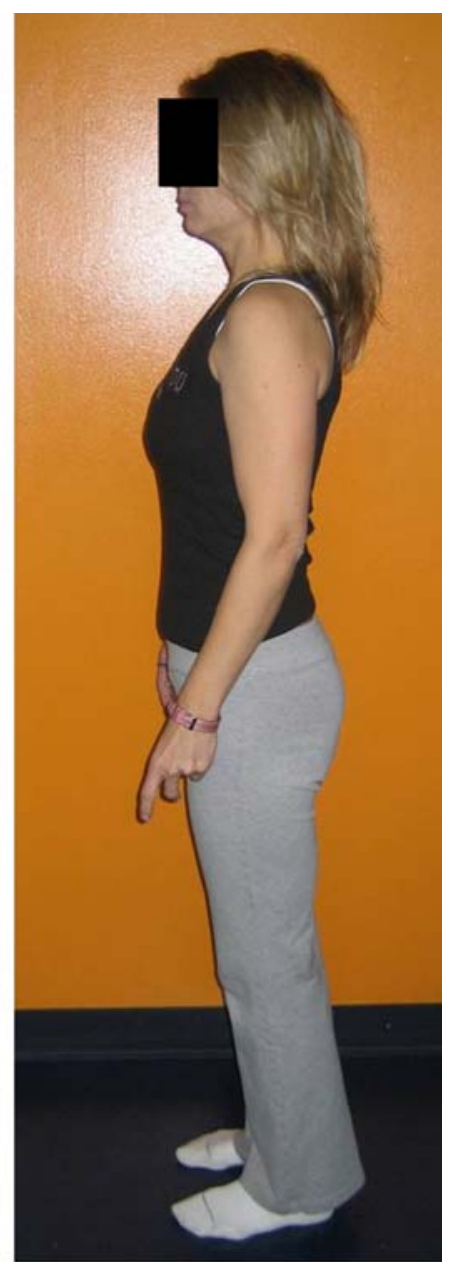

Six weeks of physical therapy
Fig. 5. The subjects' initial posture is compared to the result after 6 weeks of physical therapy 
posture and walking, but no increase in deep hip pain. Ball squats were introduced to the program, and the Reformer was removed. She was now able to exhibit good trunk control in an antigravity position therefore making this advancement appropriate. The ball squat was added to her home program.

The patient returned for a follow-up visit with her physician after the 6 weeks of physical therapy (six physical therapy sessions). Due to her success with physical therapy, a cortisone shot was not administered, and surgery was no longer a consideration. The patient was referred back to physical therapy for an additional four sessions to progress her functional core strength and continue to improve her psoas control. At the end of physical therapy, the patient was without pain for the first time since 2004. She still had intermittent clicking and discomfort in the right hip intermittently with pivoting and with wearing high heels. Her muscle function had significantly improved as evidenced by her static standing posture and her movement mechanics during gait. (Fig. 5) Upon discharge from physical therapy, the patient demonstrated increased strength in her abductors (5/5) and her abdominals (Sahrmann Level 1B), improved static, and dynamic posturing as indicated by improved recruitment of her abdominals and a decrease in her sway back posture. She was independent in a 3-day-a-week home exercise program.

\section{Discussion}

In this case, a 43-year-old female was referred for treatment of hip pain which was assumed to be caused by bilateral labral tears. Our assessment was that this patient's prior history of "low back troubles" may have contributed to her current situation. MRI revealed bilateral labral tears, but there was no evidence of hip dysplasia, femoral-acetabular impingement or history of trauma. This suggested some other mechanical or neuromuscular explanation for her hip labral tears and current hip pain. Despite a negative physical therapy lumbar scan and unremarkable PIVMs, this condition may have been generated by the prior back history, therefore yielding unilateral symptoms as a result of psoas inhibition and muscle imbalance from the initial injury. Where lumbar spine pathology is present, it has been observed that the psoas muscle changes its function. As a result of the muscle imbalances, the relationship of the lumbar spine to the hip becomes dysfunctional. [7] Over time, with the resolution of symptoms, the psoas may have begun to function, but in an abnormal manner. The result would therefore be improper neuromuscular function of the psoas and possible mechanical dysfunction modification of forces through the hip increasing the potential for hip pathology. In this case, the patient responded to therapy which initially aimed to inhibit the psoas muscle essentially removing the abnormal adaptive function followed by a program of reeducation to restore normal muscle function and strength. It appears in this case that although the primary diagnosis was a hip labral tear, the actual pain generator was a hypersensitive psoas. Therefore, by treating the soft tissue imbalances through neuromuscular reeducation and therapeutic exercise of the abdominals, pelvic floor, and hip musculature, the patient's pain completely resolved. The patient states that she still intermittently has "clicking" in the hips, but it does not cause pain.

The limitation of this case study is the lack of evidencedbased rationale behind the treatment plan. It is largely speculation and extrapolation of information based on other authors' works. Despite the success in treatment, these techniques are purely anecdotal at this time. There is a dearth of research in the area of the psoas. The success of physical therapy with this patient exemplifies how the body must be examined in its truest form, as a kinematic chain. In order for this to be accomplished, a full subjective history and a clinical system's scan must be completed prior to performing an objective exam at the localized region of injury. The etiology behind the pathology must be identified.

The second relevant factor in the treatment of this type of patient is the recognition that muscles can be inhibited and reeducated as well as stretched and strengthened. The ability of a physical therapist to recognize the factors in the malfunction and imbalance of muscles and the origin of the malfunction enables them to treat efficiently and thoroughly. Then, the manner in which the muscle has maladapted must be explored. An overactive muscle, as in this case, must first be inhibited and then functionally strengthened.

\section{References}

1. Page $P$ (2007) The Janda approach to musculoskeletal pain

2. Bullock-Saxton JE, Janda V, Bullock MI (1993) Reflex activation of gluteal muscles in walking. Spine 18(6):704-708

3. Bullock-Saxton JE, Janda V, Bullock MI (1994) The influence of ankle sprain injury on muscle activation during hip extension. Int J Sports Med 15(6):330-334

4. Cowen SM, Schache AG et al (2004) Delayed onset of transversus abdominus in long-standing groin pain. Med Sci Sports Exerc 36 (12):2040-2045

5. Panjabi MM (1992) The stabilizing system of the spine. Part I Function, dysfunction, adaptation, and enhancement. J Spinal Dis 5 (4):383-389

6. Janda V (1987) Muscles and motor control in low back pain: assessment and management. In: Twomey LT (ed) Physical therapy of the low back. Churchill Livingstone, New York, pp 253-278

7. Pettman E (2007) Lecture presented for the NAIOMT Lecture Series. Andrews University, Berrien Springs, Michigan

8. Gracovetsky SA, Iacono S (1987) Energy transfers in the spine engine. J Biomed Eng 9(2):99-114 